\title{
Energetic Electrons in Magnetosphere during Gradual Solar Energetic Particle Event Observations by Cluster
}

\author{
C.J. Xiao ${ }^{1}$, Z.Y. Pu ${ }^{2}$, H.F. Chen ${ }^{2}$, L. Xie ${ }^{2}$, Q.G. Zong ${ }^{3}$, \\ T.A. Fritz ${ }^{3}$ and P.W. Daly ${ }^{4}$ \\ ${ }^{1}$ National Astronomical Observatories, Chinese Academy of Sciences, Beijing 100012, China \\ email: cjxiao@pku.edu.cn \\ ${ }^{2}$ Institute of Space Physics and Applied Technology, Peking University,Beijing 100871, China \\ ${ }^{3}$ Center for Space Physics, Boston University, U.S.A. \\ ${ }^{4}$ Max-Planck-Institut for Aeronomie, Katlenburg-Lindau, Germany
}

\begin{abstract}
More than 40 gradual SEPs have been observed by GOES and SOHO from 2001 to 2003. During 12 SEPs of all these events, energetic electron flux enhancements with energies from $38 \mathrm{keV}$ to $337 \mathrm{keV}$ were observed by RAPID onboard Cluster spacecraft. During these 12 events the variation of the energetic electron flux measured by RAPID/Cluster was closely associated to the variation of the solar energetic proton flux. The observed energetic electron flux was independent on the location of Cluster in the magnetosphere and the background level of magnetospheric electrons (even when the Cluster spacecraft was crossing the magnetopause, the plasma sheet and the low latitude boundary layer). The similar variation of the enhancement of energetic electron flux has also been observed by POLAR and Geotail in some of these events. In some of these events, the electron measurement of RAPID/Cluster will not be contaminated by the SEP protons.
\end{abstract}

Keywords. Sun: coronal mass ejections (CMEs), particle emission

\section{Introduction}

The gradual solar energetic particle events (SEPs) in association with coronal mass ejections (CME) have important consequences for space weather. The geoeffectiveness of SEPs is one of key issues in space science research (Reames (1999) and references herein).

The Cluster mission is designed to study the small-scale structures and multi-scale dynamics in the magnetosphere(Escoubet Schmidt \& Goldstein (1997)). The RAPID spectrometer (Research with Adaptive Particle Imaging Detectors) for the Cluster mission is an advanced particle detector for the measurement of energetic particles in the energy range from $20-400 \mathrm{keV}$ for electrons, $40 \mathrm{keV}-4000 \mathrm{keV}$ for proton and other heavier ions (Wilken, et al. (1997)).

More than 40 gradual SEPs have been observed by GOES and SOHO from 2001 to 2003. During 12 SEPs of all these events, energetic electron flux enhancement were observed by RAPID/Cluster. During these 12 events the variation of the energetic electron flux measured by RAPID/Cluster was closely associated to the solar energetic proton flux variation. The observed energetic electron flux was not affected by the location of Cluster in the magnetosphere and the background level of magnetospheric electrons (even when the Cluster spacecraft were crossing the magnetopause, the plasma sheet and the low latitude boundary layer). The similar variation of the enhancement of energetic electron flux has also been observed by POLAR and Geotail in some of these events. 


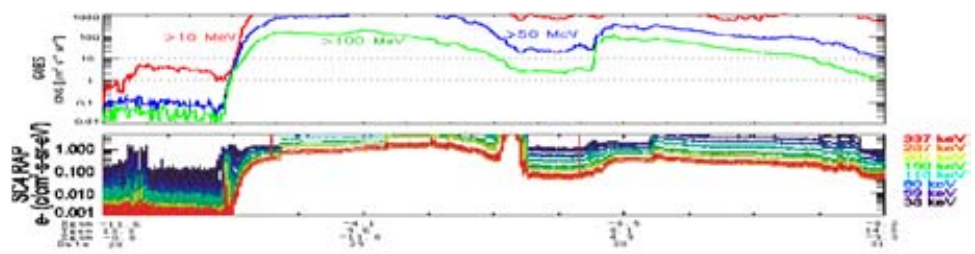

Figure 1. Observations of the energetic particles on Oct. 28-31, 2003 event.top:The integral flux of energetic ions measured by GOES; bottom: the differential flux of energetic electrons measured by Cluster and the position of Cluster in GSM coordinate system.

\section{Oct. 28-31, 2003 event}

From Oct. 28 11:00 to Oct. 31, 20:00 UT, the Cluster spacecraft traversed the magnetosheath, magnetopause, plasmasphere and radiation belts. The flux of energetic electrons observed by RAPID/Cluster was unusually high. The enhancement lasted during the SEPs (Figure 1), while the flux of energetic ions observed by RAPID-IIMS did not show any special change. The similar results were measured by the Geotail, ACE and WIND spacecraft. The variations of the energetic electron flux measured by those satellites were all closely associated to the variations of solar energetic proton flux measured by the GOES spacecraft in the geosynchronous orbit. The observed energetic electron flux was independent on the locations of Cluster or Geotail in the magnetosphere and the background level of the magnetospheric electrons.

\section{Discussions and Conclusions}

Electrons, even with the energy as high as $1 \mathrm{MeV}$, are very difficult to entry the magnetosphere by directly penetrating across the magnetopause. Furthermore, the hard x-ray or $\gamma$-ray burst could not keep to contaminate the energetic electron measurement for several days. Therefore the question arises: whether or not the electron flux enhancements observed by RAPID/Cluster were contaminated by the solar energetic protons?

It is known that the solid state detectors of the RAPID/Cluster can be shielded from the solar energetic protons with the energy lower than $100 \mathrm{MeV}$ (Wilken, et al. (1997)), while during some events the fluxes of solar energetic protons with energy more than $50 \mathrm{MeV}$ (observed by GOES) were two-order smaller than the fluxes of energetic electrons. During the Mar. 23, 2002 event, while the flux of energetic protons higher than $10 \mathrm{MeV}$ maintained on the background level, the energetic electrons flux enhancements observed by RAPID/Cluster. So the solar energetic protons can not be the contamination source in these events.

In a summary, at lease in some of these 12 SEPs, neither solar energetic protons nor the $\mathrm{x}$-ray or $\gamma$-ray bursts can be the main contamination source of the electron measurement by RAPID/Cluster. The other physical reasons need to be discussion further.

\section{Acknowledgements}

This work is supported by the Chinese DSP-Cluster Science Team and by the CNSF Key Project 40390150 and Chinese Fundamental Research Project G200000784.

\section{References}

Escoubet, C.P., Schmidt, R., and Goldstein M.L. 1997 Space Sci. Rev. 79, 11

Reames, D.V. 1999, Space Sci. Rev. 90, 413

Wilken, B., Axford, W.I., and Daglis, I. et al. 1997, Space Sci. Rev. 79, 399 